\title{
Spontaneous spinal epidural hematoma during pregnancy
}

Hematoma extradural espinhal espontâneo durante a gravidez

Shigeki Matsubara

\section{Dear Editor,}

Braga et al. ${ }^{1}$ described the conservative treatment of spontaneous spinal extradural hematoma (SSEH). I would like neurologists to pay more attention to SSEH and pregnancy. I have three clarifications.

First, pregnancy is a risk factor for SSEH. Recently, we reported a woman with twin pregnancy suffering SSEH at the $16^{\text {th }}$ week of gestation ${ }^{2}$. To our knowledge, 14 cases of SSEH during pregnancy have been reported ${ }^{2,3}$ : decompression surgeries were performed in all cases. Both veins and arteries can be candidates of origin for SSEH. In the former case, pregnancy increases abdominal pressure, which may lead to a rise in the epidural venous pressure, possibly leading to the rupture of a venous wall. In the latter case, how pregnancy affects it is unclear.

Second, short lag time between symptom onset and surgery may not always guarantee recovery, which is important not only medically but also judicially. Surgical decompression within 12 hours of the symptoms' onset has been generally recommended ${ }^{1}$. Focusing on the topic to SSEH during pregnancy, of 14, 3 had complete neurological recovery while the remaining 11 did not. Lag time was a median of 24 (range: 8 to 31 ) and 15 (7 to 36) hours, respectively, showing no difference. In the present case, it was nine hours; however, the patient still could not walk. Braga et al. ${ }^{1}$ stated: "controversy exists regarding the appropriate time for surgery" Expectant management also led to good recovery in cases with mild neurological deficits ${ }^{1}$. Prognosis may also be influenced by rehabilitation.

Third, a rehabilitation program should be established for pregnant women. Rehabilitation after surgery or expectant management may be important for good neurological recovery; however, pregnant patients provide specific concerns. If SSEH occurs in early pregnancy week, "evacuation and continue pregnancy" strategy may be employed, which may require a view toward subsequent rehabilitation. Of $14 \mathrm{SSEH}$ during pregnancy, cesarean section was performed at the time of evacuation in 9, while pregnancy was continued after evacuation in 5 cases, depending on the gestational weeks: SSEH occurred at a median of 37 (range 32 to 41) and 24 (16 to 34) weeks, respectively. Of the latter, four gave term birth, while our patient yielded preterm delivery for whom rehabilitation was started soon after surgery. Although we were cautious about preterm labor and thus closely monitored uterine contractions, she gave birth to a preterm infant at the $29^{\text {th }}$ week; the rehabilitation may be one possible culprit for uterine contractions. An effective rehabilitation program least inducing uterine contractions may benefit pregnant women not only in this specific condition, but also for those requiring rehabilitation, and not sufficiently given it for fear of uterine contractions. Cooperative effort of clinical neurologists and obstetricians may be needed to establish a rehabilitation program for pregnant women.

\section{References}

1. Braga MH,Brandão RA, Carvalho GT, Santos CD, Abreu MS. Conservative treatment of large spontaneous spinal extradural hematoma. Arq Neuropsiquiatr 2010;68:132-134.

2. Matsubara S, Inoue H, Takamura K, et al. Spontaneous spinal epidural hematoma at the $16^{\text {th }}$ week of a twin pregnancy. J Obstet Gynaecol Res 2011. doi: 10.1111/j.1447-0756.2010.01522.x. [Epub ahead of print].
3. Sarubbo S, Garofano F, Maida G, Fainardi E, Granieri E, Cavallo MA.
Spontaneous and idiopathic chronic spinal epidural hematoma: two
case reports and review of the literature. Eur Spine J 2009;18:10551061. 